\title{
Determinants of Manual Dexterity in Young Adults
}

\author{
By \\ Ryan C. Price \\ B.S., United States Air Force Academy, 2008
}

\author{
A thesis submitted to the \\ Faculty of the Graduate School of the \\ University of Colorado, in partial fulfillment \\ of the requirement for the degree of \\ Master of Science \\ Department of Integrative Physiology
}

2014 
This thesis entitled:

"Determinants of Manual Dexterity in Young Adults"

written by Ryan C. Price

has been approved for the Department of Integrative Physiology.

Roger M. Enoka, Ph.D.

David E. Sherwood, Ph.D.

Alaa A. Ahmed, Ph.D.

Date 1 December 2014

The final copy of this thesis has been examined by the signatories, and we find that both the content and the form meet acceptable presentation standards of scholarly work in the above mentioned discipline.

IRB protocol number: $14-0356$

The views expressed in this thesis are those of the author and do not reflect the official policy or position of the United States Air Force, Department of Defense, or the U.S. Government. 
Price, Ryan C (M.S. Integrative Physiology)

Determinants of Manual Dexterity in Young Adults

Thesis directed by Roger M. Enoka, Ph.D.

\begin{abstract}
The time to complete a test of manual dexterity (Grooved Pegboard Test) increases with advancing age due to changes in force steadiness, muscle strength, and cognitive function. However, the relative significance of these predictors among healthy young adults remains unknown. PURPOSE: To identify predictor variables that can explain the variance in the time that it takes young adults to complete the Grooved Pegboard Test. It was hypothesized that an expanded set of force steadiness tests, as well an assessment of cognitive function, would predict a significant amount of the variance in pegboard times. METHODS: Twenty-seven young adults (23.8 \pm 4.0 years) performed three trials of the Grooved Pegboard Test with the right (dominant) hand. Strength was measured during handgrip, wrist extension, pinch, and index finger abduction tasks. Force steadiness measurements were made at $5 \%$ and $10 \%$ of maximum during traditional one-joint tests (wrist extension, index finger abduction, and pinch) as well as during novel two-joint tests (wrist extension combined with either index finger abduction or pinch). Cognitive function was assessed using a test of reaction time. RESULTS: 1) Time to complete the Grooved Pegboard Test was $52.5 \pm 4.8 \mathrm{~s}$ (mean \pm SD). 2) Force steadiness was worse for two-joint tests $(3.78 \pm 1.23 \%)$ than one-joint tests $(2.50 \pm 0.71 \%, P<0.001)$. 3$)$ Force steadiness was negatively correlated with pegboard times in 13 of 14 tests (e.g., wrist extension at $10 \%, r=-0.452, P<0.01$ ). 4) Pegboard times were not significantly correlation with reaction time or any measure of strength. 5) Multiple regression analysis explained $37 \%$ of the variance in the time to complete the Grooved Pegboard Test using a model containing a single one-joint test and two of the two-joint force steadiness tests of force steadiness. CONCLUSIONS: The use of an expanded set of steadiness tests significantly improved the ability to predict pegboard times among young adults. Nonetheless, a substantial amount of the variance in the pegboard times for young adults remains unexplained.
\end{abstract}




\section{Acknowledgements}

None of my efforts would be possible without the support and sponsorship of the Air Force Institute of Technology. Particular thanks goes to the U.S. Air Force Academy Faculty Pipeline Program and the Department of Biology for enabling this opportunity.

I would also like to thank my advisor, Professor Roger Enoka, for giving me the opportunity to join his Neurophysiology of Movement Laboratory and most importantly for his guidance, mentorship, and trust during my time at the University of Colorado. Additionally, I would like to thank the other committee members, Professors David Sherwood and Alaa Ahmed, for donating their time and energy into the review of this thesis.

I also owe my fellow laboratory members thanks; particularly Awad Almuklass for his tireless work in every aspect of this project. Awad was a great teammate and his input and ideas were essential to the successful completion of this manuscript. I similarly owe thanks to the rest of my lab mates for their assistance in editing, brainstorming, testing, and most of all, for making my time the lab an incredible and rewarding experience.

Finally, I want to thank my fiancé, Mariam, for her tireless support, boundless energy, and continuous love. 


\section{Contents}

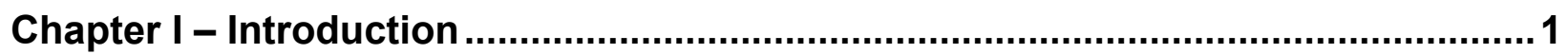

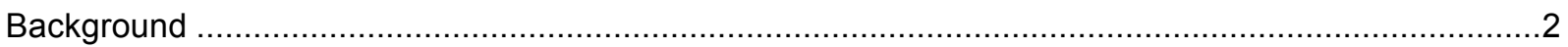

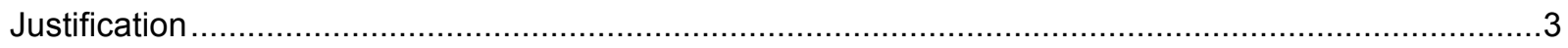

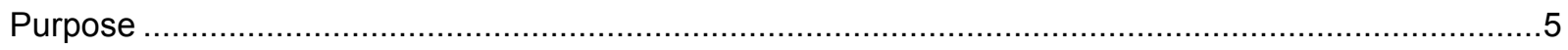

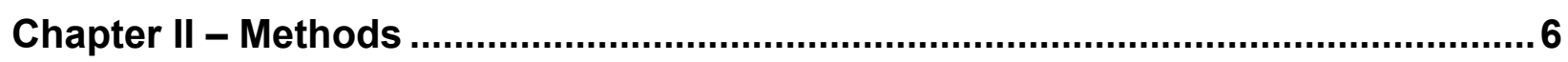

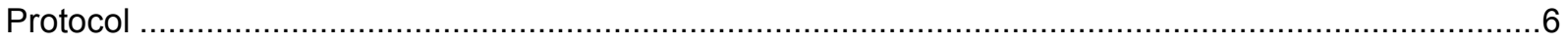

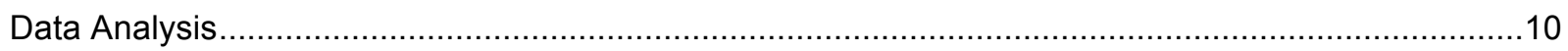

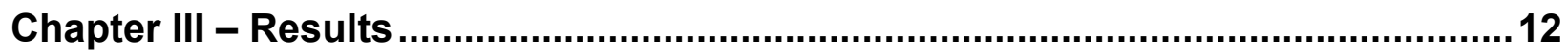

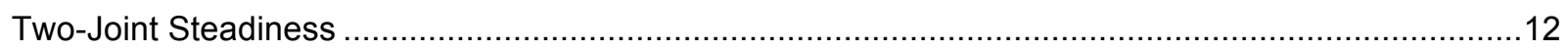

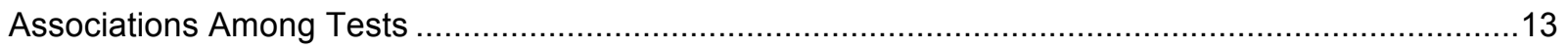

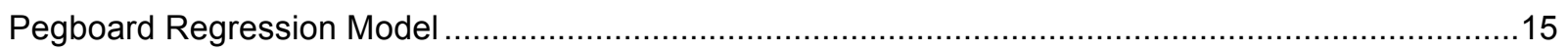

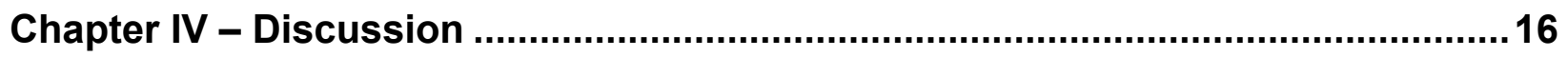

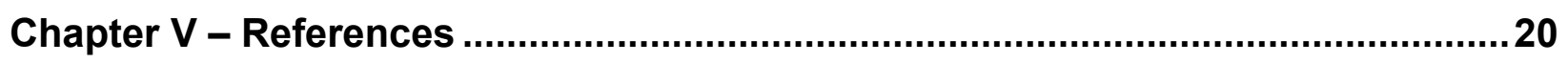




\section{Figures}

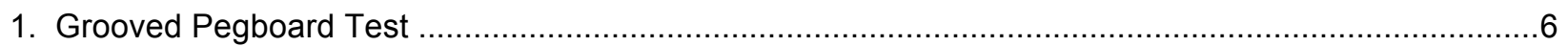

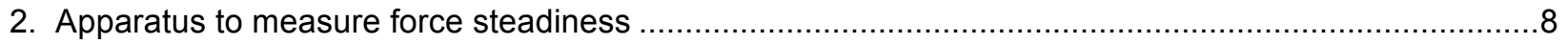

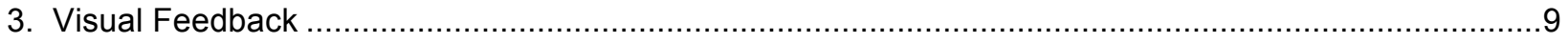

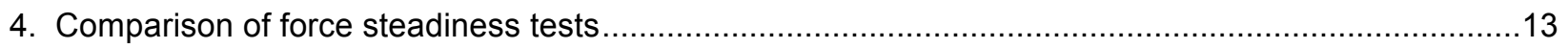

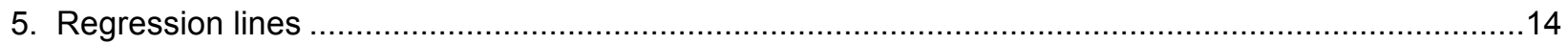

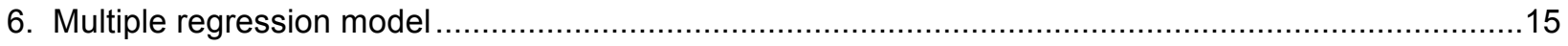




\section{Tables}

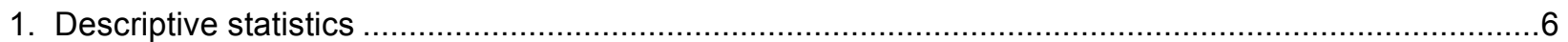

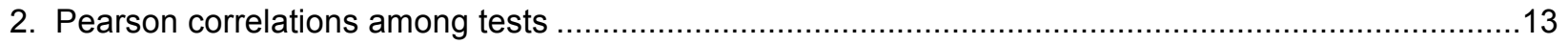

3. Pearson correlations between predictor variables and pegboard time ...........................................14

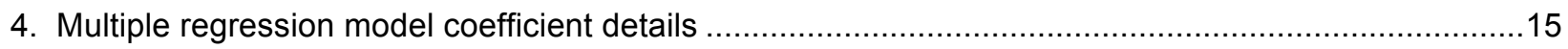

5. Descriptive statistics of young adult subjects from Marmon et al. (2011a) ....................................16 


\section{Chapter I - Introduction}

Grasping and manipulating actions are coordinated and controlled by the integration of the motor and musculoskeletal systems. The cooperative interaction of these systems enables successful motor control, which can be characterized by such properties as muscle strength, force steadiness, training status, tactile sensibility, mental acuity, level of motivation, and visuomotor acuity (Backman et al., 1992; Jeannerod et al., 1995; Birznieks, 2003; Ashendorf et al., 2009; Marmon et al., 2011a). The relative significance of these properties in the control of movement has been widely investigated by assessing their influence on manual dexterity, which is broadly defined as motor control of the hands and fingers. Dexterity is a central component of hand function as it is expressed in everyday tasks such as cooking and writing, and has been correlated with job proficiency, disease progression, and mortality (Yancosek and Howell, 2009; Buchman et al., 2007; Lundergan et al., 2007; Reuben et al., 2013; Bezdicek et al., 2014). As such, manual dexterity has both public health and scientific importance and it serves as a meaningful tool for assessing neurological health and function across the lifespan (Reuben et al., 2013).

Results from recent aging research indicate that a moderate amount of the variance in the time to complete a test of manual dexterity (Grooved Pegboard Test) can be explained by measures of finger steadiness and handgrip strength (Marmon et al., 2011a). Moreover, there is a significant association between Grooved Pegboard Test performance and cognitive function (Ashendorf et al., 2009). Accordingly, force steadiness, handgrip strength, and cognitive function can explain a significant amount of the variance in time to complete the Grooved Pegboard Test among young, middle-aged, and old adults. However, the relative significance of these predictors among healthy young adults remains unknown. The current study sought to address this gap in knowledge by examining both known and novel explanatory variables for the variance of Grooved Pegboard times of young adults.

Traditionally, force steadiness is measured during a single action that is produced around a single, experimentally isolated joint, such as the abduction force exerted by the index finger about the metacarpophalangeal joint. However, the anatomical specialization and evolution of the hand, which has been connected to human evolutionary success, is likely driven by development of tool use (Young, 2003). Thus, experimental restrictions of such isolated actions calls into question the ecological validity of 
traditional one-joint tests. Therefore, the force steadiness tests in the current study included a series of novel two-joint actions that measure the steadiness of two forces concurrently, such as during a pinch and wrist extension task, which are a grouping of actions that are fundamentally involved in the pegboard task. It was hypothesized that these more ecologically meaningful tests, as well as the assessment of cognitive processing speed, would predict a significant amount of the variance in the time that it takes young adults to complete the Grooved Pegboard Test.

\section{Background}

Force Steadiness. When an individual attempts to exert a constant force, the generated force naturally fluctuates about an average value (Loscher and Gallasch, 1993; Tracy and Enoka, 2002). As the level of force production increases, the absolute variability of the force (standard deviation) also increases, whereas the normalized variability (coefficient of variation, CV) decreases (Galganski et al., 1993).

The motor system's production of force by a muscle depends on the number of individual motor units recruited and the rate at which they discharge action potentials (Enoka et al., 2003). During voluntary contractions, motor neurons receive common synaptic contributions from both descending and peripheral inputs (Semmler et al., 1997). This results in a phenomenon called common drive, or the simultaneous fluctuation of discharge rates among multiple motor units (DeLuca et al. 1982). Common drive is ultimately responsible for force fluctuations during constant-force contractions, and can be influenced by the visual demands of the task, type of muscle contraction, properties of the motor units recruited, and the physical activity level of the individual (Enoka et al., 2003; Negro et al., 2009; Marmon and Enoka, 2010).

Grooved Pegboard Test. The Grooved Pegboard Test (Lafayette Instrument Company, Inc.) was designed to evaluate a number of psychomotor functions including hand-eye-coordination, motor speed, and manual dexterity (Kløve, 1963; Strauss et al., 2006). The timed test requires subjects to insert keyshaped pegs into 25 holes of varying orientation as quickly as possible. Recently, the National Institutes of Health (NIH) established a Toolbox for the Assessment of Neurological and Behavioral Function in an 
effort to provide a reliable set of brief, yet comprehensive, assessments to evaluate motor, cognitive, sensory, and emotional function across the lifespan (Reuben et al., 2013). Dexterity was included among the various motor subdomains and the Grooved Pegboard Test was recommended for possible inclusion in the Toolbox Supplemental Measures since it has the capacity of assessing high dexterity function (Wang et al., 2011; Reuben et al., 2013).

Cognition. Reaction time tests can estimate cognitive demand by measuring the information processing speed of the central nervous system. The literature on reaction time distinguishes two time periods: premotor time and motor time. Premotor time represents the time from stimulus onset to muscle activation, whereas motor time represents the time from muscle activation to the moment at which the movement triggers the desired motor response. Reaction times increase as a task becomes more difficult, and this increase is typically due to longer premotor times because of the increase in cognitive demand (Weiss, 1965; Clarkson, 1978; Fozard et al., 1994).

\section{Justification}

Force Steadiness Tests. Marmon et al. (2011a) investigated the associations among strength, force steadiness, and functional measures in young, middle-aged, and old adults. They found that $36 \%$ of the variance in Grooved Pegboard Test times was explained by force steadiness during index finger abduction $(r=0.57)$ and grip strength $(r=-0.34)$. In a subsequent study, Marmon et al. (2011b) found

that practice reduced the time it took older adults to complete the Grooved Pegboard Test and that faster times were associated with an improvement in force steadiness of intrinsic hand muscle. These studies show that manual dexterity is associated with both the quality (force steadiness) and quantity (muscle strength) of motor unit activity. By demonstrating the discriminatory power of force steadiness and hand strength, these findings suggests that other measures of force steadiness and strength may be able to explain a larger amount of the variance in pegboard times.

Force steadiness tests typically limit the number of muscles involved in the task in order to examine the association between the activation signals delivered to the muscle and the net muscle force (Galganski et al., 1993; Marmon et al., 2011a). For example, force steadiness during index finger 
abduction involves only the action of the first dorsal interosseus (FDI) muscle about the metacarpophalangeal joint. However, such one-joint actions rarely occur during activities of daily living. Despite this constraint, it is surprising that performance of a relatively simple isometric contraction can predict a moderate amount of the variance in the time it takes to complete the test of manual dexterity, especially considering that the Grooved Pegboard Test requires exquisite control of motor unit activity.

Two-joint tests examine the force steadiness during actions performed concurrently about two independent axes. Such tests can require individuals to control both an intrinsic hand action (pinch or index finger abduction) and a related extrinsic hand action (wrist extension). Research on grasp-and-lift actions utilizes a similar setup that combines a pinch force with a lift force in order to assess digit forces, grasp coordination, and the inputs of afferent information by examining the coupling of pinch and lift forces (Westling and Johansson, 1984; Per and Johansson, 1997; Monzée et al., 2014). Given the strength of one-joint tests, it is theorized that two-joint tasks, as in the grip-and-lift studies, will offer a more ecologically valid and useful measure of force steadiness.

Cognition. Motor control is influenced by cognitive function. Ashendorf et al. (2009) found that $21 \%$ of the variance in time needed to complete the Grooved Pegboard Test could be explained by cognitive tests. Assessment of cognitive processing speed via reaction time tests can be accomplished using either simple reaction time or choice reaction time tests. Choice reaction time tests are more challenging in that uncertainty is introduced into the test by the use of multiple stimuli requiring a variety of responses (Logan, 1980). For the purposes of the current study, a choice reaction time test (hereafter referred to as a reaction time test) was implemented in the form of a computerized model of Fitts' Law (Goldberg et al., 2013). This test offers a method for assessing reaction time by presenting the individual with targets of varying difficulty that alter in both size and location. Fitts' Law states that movement speed decreases as movement difficulty increases (Fitts, 1954). Consequently, smaller, more distant targets are more difficult to reach than larger, closer targets and the resulting movement speed declines (Goggin and Meeuwsen, 1992; Ketcham et al., 2002). Such a test mirrors the challenges associated with performing the Grooved Pegboard Test, as the locations of both the pegs and the holes change 
progressively throughout the test. Therefore, tests of reaction time are likely associated with the time it takes to complete the Grooved Pegboard Test.

\section{Purpose}

The purpose of this study was to identify predictor variables that can explain the variance in time it takes young adults to complete the Grooved Pegboard Test. The primary outcomes included measurements of grip strength and force steadiness during one- and two-joint force steadiness tests. Additionally, reaction time was used to assess the association between manual dexterity and cognitive function. It was hypothesized that the strongest predictors of variance in the time it takes young adults to complete the Grooved Pegboard Test would be reaction time and force steadiness during two-joint actions. 


\section{Chapter II - Methods}

Twenty-seven right-handed young adults (18-34 years, 13 women, Table 1) met the inclusion criteria and participated in the study after informed consent was obtained. All subjects were free from neurological disease and were not taking any medications known to influence neuromuscular or cognitive function. Additionally, all subjects were screened for musculoskeletal abnormalities that could influence upper limb function and were unfamiliar with the experimental protocol. The Institutional Review Board at the University of Colorado Boulder approved the protocol.

\section{Protocol}

Subjects completed the Edinburgh Handedness Inventory - Short Form (Veale, 2014). Subjects who scored in the range of 60 to 100 were confirmed as righthanded and included in the study. The subjects then performed several functional and force steadiness tests. The total time required to complete the protocol was

\begin{tabular}{lc} 
& Total \\
\hline $\mathrm{N}$ & 27 \\
Age $(\mathrm{yrs})$ & $23.8 \pm 4.0$ \\
Height $(\mathrm{cm})$ & $174.2 \pm 9.9$ \\
Mass $(\mathrm{kg})$ & $71.2 \pm 14.4$ \\
Pegboard Time $(\mathrm{s})$ & $52.5 \pm 4.8$ \\
Reaction Time $(\mathrm{ms})$ & $925.5 \pm 79.7$ \\
Grip Strength $(\mathrm{N})$ & $430.2 \pm 101.1$ \\
\hline
\end{tabular}

Table 1. Descriptive statistics. Values represent mean \pm SD.

approximately two hours.

Grooved Pegboard Test. This test utilized the Grooved Pegboard (Figure 1) and required subjects to place metal pegs into 25 holes on a board as quickly as possible using the right hand. The holes resembled keyholes arranged in a $5 \times 5$ matrix with varying orientation across the board. The pegs resembled keys with a raised ridge and only through manipulation of a peg's orientation could it be successfully inserted into the

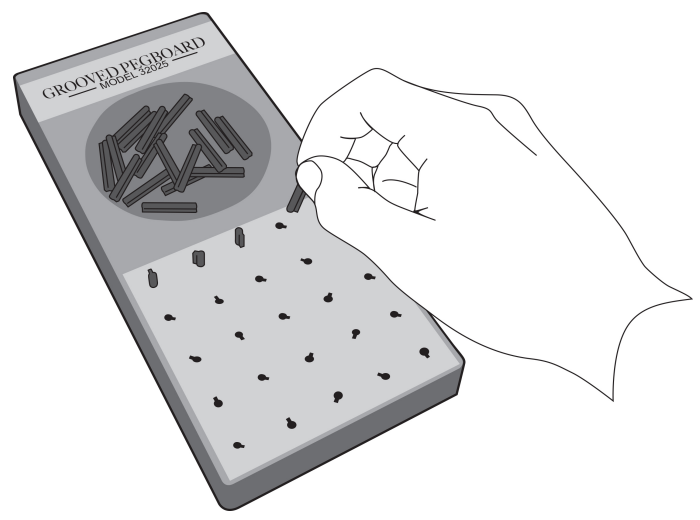

Figure 1. Grooved Pegboard Test. Manual dexterity is quantified as the average time needed to insert 25 pegs during three trials with the right (dominant) hand. Note the orientation of the holes that vary across the board requiring the in-hand rotation and fine manipulation of the pegs. hole. Subjects were instructed to fill the board one row at a time, from left to right and top to bottom. This test was performed three times and the time required to 
complete each trial was recorded. The average time to complete all three trials was used to quantify manual dexterity.

Reaction Time Test. This test utilized a feature of a larger online Java Applet that has been used to assess Fitt's Law (Goldberg et al., 2013). The test required participants to manipulate the position of a cursor on a computer screen with a mouse to click on 25 circular targets of varying size and location as they randomly appeared on the screen. All subjects were presented with the same 25 targets but in varying order and instructed to perform this test as quickly as possible. The time elapsed from target presentation to successfully clicking on the target was averaged and recorded. Subjects completed one practice trial and one experiential trial. The time obtained during the experimental trial was used to quantify reaction time.

Handgrip Strength. Maximal isometric grip strength of the right hand was recorded as the peak force achieved during a maximal voluntary contraction (MVC) using a hand dynamometer (Hydraulic Hand Dynamometer, Baseline Evaluation Instruments, White Plains, NY). Subjects were comfortably seated without arm support and instructed to maintain $90^{\circ}$ of elbow flexion and full shoulder adduction during each MVC trial. To ensure the reliability of the maximal values, MVC measurements were repeated with $30 \mathrm{~s}$ of rest between measurements until the peak forces of two MVC trials were within $5 \%$.

Force Steadiness. An apparatus (Figure 2) was fabricated to measure force steadiness during actions about one-axis (one-joint tasks) or two-axes (two-joint tasks). Two configurations were developed: one designed for index finger abduction (Figure 2A) and a second for pinch (Figure 2B). Both configurations also enabled the measurement of wrist extension forces. One-axis tasks measured force steadiness during wrist extension, index finger abduction, or pinch tasks only. Two-axis tasks detected force steadiness when wrist extension was combined with either index finger abduction or pinch. Each of the force steadiness tests used force targets of $5 \%$ and $10 \%$ of MVC during the force-matching tasks.

Subjects were seated in a chair with the right shoulder abducted to approximately $90^{\circ}$, the elbow flexed to $90^{\circ}$, and the forearm secured to a support using polyester straps and foam cushioning. Bilateral 
shoulder straps and a lap belt were used to restrict shoulder and torso movement. A cushioned bar attached to a force sensor (0.0056 V/N, JR3 Model 45E15A-U760-A, Woodland, CA) rested on the back of the right hand directly above the heads of metacarpals two through five and measured the upward force produced during an isometric with the wrist extensors. The index finger abduction configuration (Figure 2A) involved an additional platform placed below the phalanges of the right hand, which enabled the abduction force exerted by the index finger about the metacarpophalangeal joint to be measured. The index finger was fitted with a custom made orthotic that contacted a button load cell $(0.049 \mathrm{~V} / \mathrm{N}$, Futek Model LLB130, Irvine, CA) at the radial border of the proximal interphalangeal joint. The pinch configuration (Figure $2 \mathrm{~B}$ ) required the subject to hold a similar, but unrestrained, button load cell between the index finger and thumb pads, which was used to measure the pinch force.

The force data were low-pass filtered at $50 \mathrm{~Hz}$ with a Butterworth filter (V75-48 High Performance Bandpass Filter, Coulbourn Instruments, Whitehall, PA). Data were sampled at $2000 \mathrm{~Hz}$ using an analogto-digital converter (Power 1401, Cambridge Electronic Design, Cambridge, UK). All data collection took place within the Spike2 data acquisition system (Version 5.20, Cambridge Electronic Design, Cambridge, UK) and stored for offline analysis.

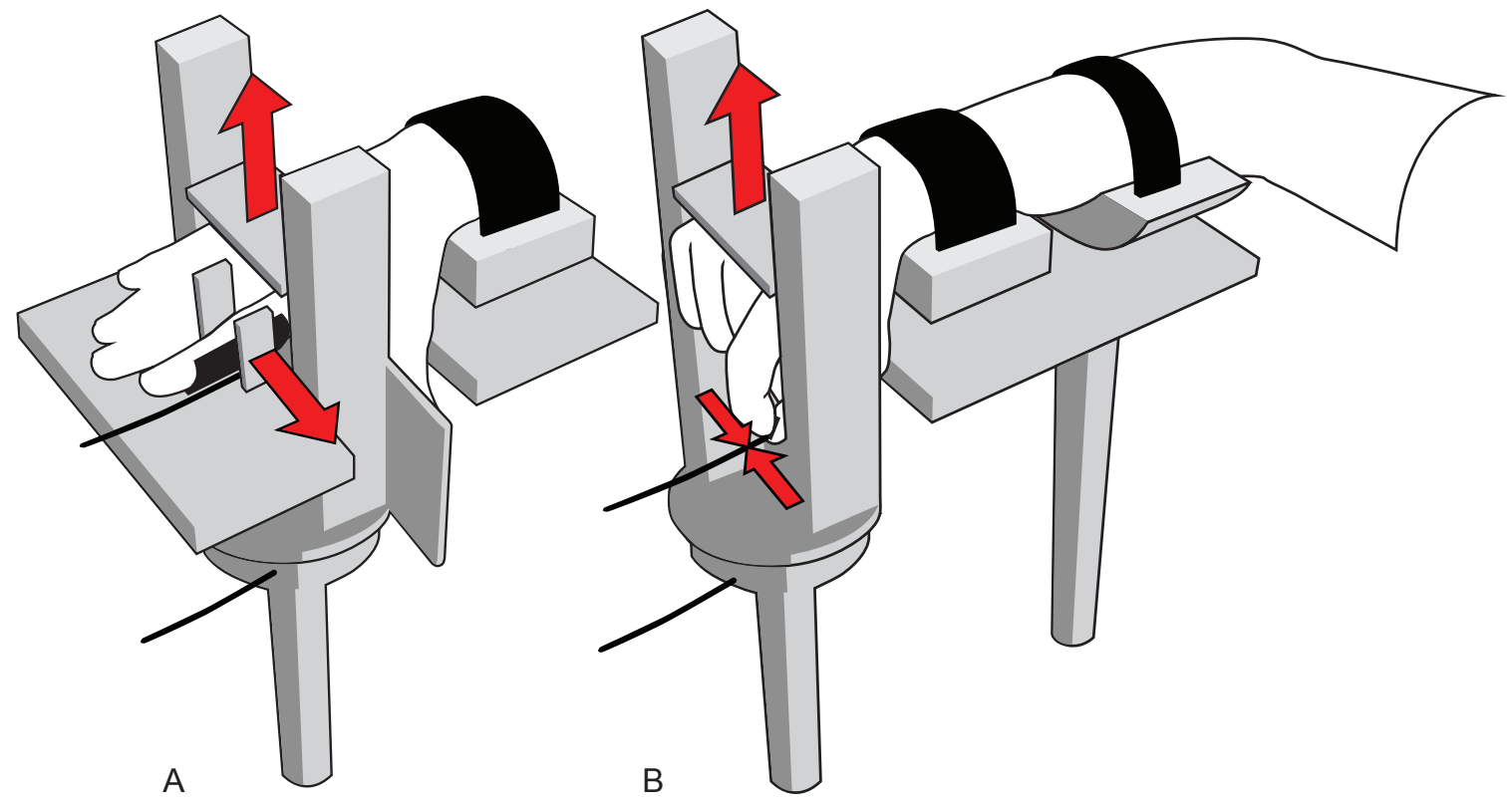

Figure 2. Apparatus to measure force steadiness during one- and two-joint steady isometric contractions. The measured forces were index finger abduction $(A)$, pinch $(B)$, and wrist extension $(A$ and $B)$. 
Maximal Voluntary Contractions. Peak forces were measured during MVCs in wrist extension, index finger abduction, and pinch tasks. Subjects were instructed to increase force from rest to maximum gradually during a $3 \mathrm{~s}$ period and then to hold a maximal force for $2 \mathrm{~s}$. To ensure the reliability of the MVC force, measurements were repeated with $30 \mathrm{~s}$ of rest until the peak forces of two MVC trials were within $5 \%$.

Visual Feedback. Each force steadiness test required subjects to perform submaximal, isometric contractions. These force-matching tasks required the display of both one and two independent forces concurrently (Figure 3A). All feedback was displayed on a computer monitor (19 inch Dell, $1280 \times 1024$, $60 \mathrm{~Hz}$ ) placed 1.5 meters in front of the subject. Visual gain was normalized to $0.66 \% \mathrm{MVC} / \mathrm{cm}$ in the $\mathrm{y}$ axis and $0.53 \% \mathrm{MVC} / \mathrm{cm}$ in the $\mathrm{x}$-axis. A force indicator (red crosshairs) was used to convey the magnitude of the forces being exerted by the subject. Forces produced by wrist extension were displayed as positive, vertical deviations of the indicator, whereas forces produced by pinch (or index finger abduction) were displayed as leftward, horizontal deviations of the indicator. The visual feedback included either one or two targets depending on the task (Figure 3A shows two targets). Two-joint tests utilized a horizontal line as the target for wrist extension and a vertical line as the target for the index finger abduction or pinch. One-joint tests required only a single target line, either horizontal or vertical depending on the task.

A

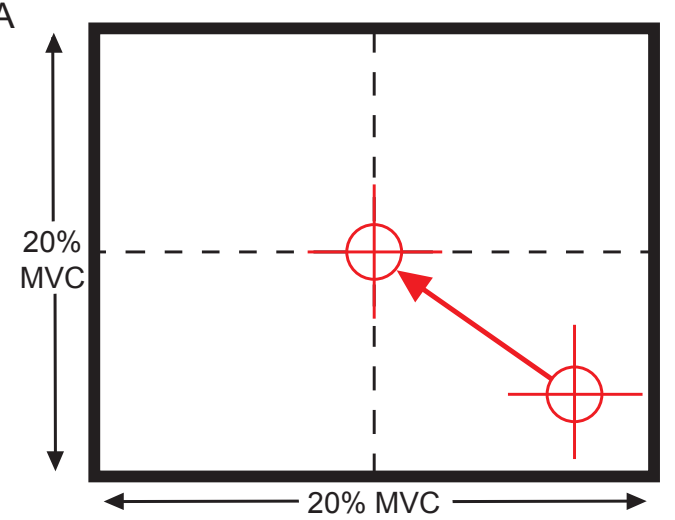

B

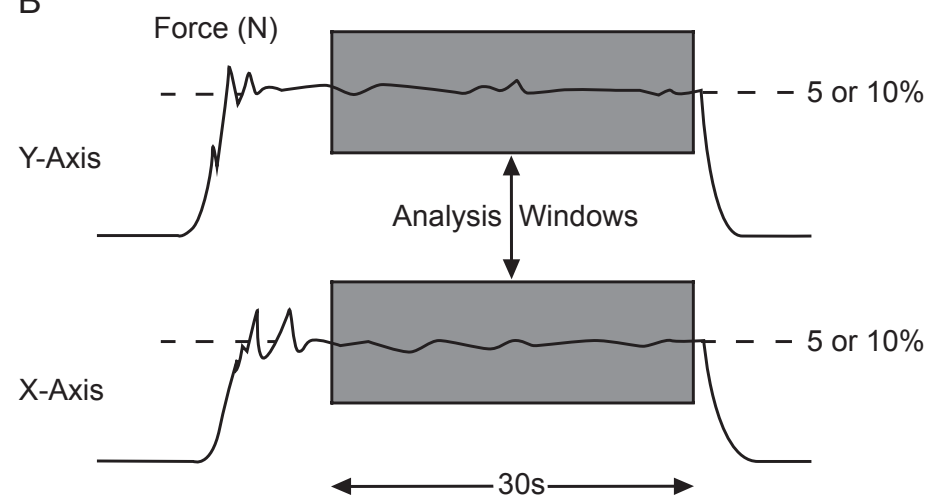

Figure 3. Visual feedback of two target forces during two-joint steadiness tests. (A) Forces were displayed in an $x-y$ plot. Wrist extension forces produced a positive vertical deflection of the indicator (crosshairs shown in red), whereas pinch or index finger abduction forces produced a leftward horizontal deflection of the indicator. The dashed lines represent separate target forces. (B) Two force traces measured concurrently. The shaded area indicates the 30-s window during which steadiness was calculated. 
One-Joint Tests: Wrist Extension, Pinch \& Index Finger Abduction. A target line representing 5\% or $10 \%$ of MVC force was displayed using either a horizontal (wrist extension) or vertical (pinch and index finger abduction) target line on the monitor. The subject was instructed to move the indicator to match the target line as quickly and as accurately as possible during two, 30-s experimental trials with $30 \mathrm{~s}$ of rest between trials. Subsequently, the same procedures were repeated for the other target force. The $5 \%$ and $10 \%$ target force trials were performed in a randomized order.

Two-Joint Tests: Wrist Extension and Pinch \& Wrist Extension and Abduction. Two target lines were displayed, as described for the one-joint tests, but at the same time. The intersection of the horizontal and vertical lines (Figure 3B) represented the target for the two-joint test, which subjects were instructed to match with the indicator as quickly and as accurately as possible during two $30-\mathrm{s}$ experimental trials with $30 \mathrm{~s}$ of rest between trials. Subsequently, the same procedures were repeated for the other target force. The $5 \%$ and $10 \%$ target force trials were performed in a randomized order.

\section{Data Analysis}

Force Steadiness. Offline processing of force data was performed during selected 30-s windows (Figure 3B). Force steadiness was quantified as the coefficient of variation (CV $=$ [standard deviation / mean force] $\times 100$ ) for each force and was averaged over two 30 -s trials.

Statistical Analysis. A sample size of 25 young adults was estimated from results of Marmon et al. (2011a), which corresponds to an effect size (Cohen's d) of 0.86 and achieves a statistical power of $80 \%$ at an alpha level of 0.05 . An additional two subjects were recruited as a precaution for technical difficulties.

Paired t-tests compared data between one-joint and two-joint tests as well as the difference between similar tests at $5 \%$ and $10 \%$. Pearson correlations were used to assess potential associations among the outcome variables. All functional and strength tests were normally distributed as confirmed by a Shapiro-Wilk's test and a visual inspection of the histograms, normal Q-Q plots, and box plots. In contrast, force steadiness measures tended to be slightly skewed and kurtotic and did not follow a normal 
distribution. As a result, non-parametric tests verified the significance found in both the paired t-tests and the Pearson correlations.

A backwards, stepwise, multiple regression method was used to calculate a model that could predict the variance in the times to complete the Grooved Pegboard Test. During analysis of the 270 force steadiness trials, six were excluded due to errors caused by external factors. As a result, one subject was excluded $(N=26)$ from the regression analysis. Multicollinearity was estimated with variance inflation factor (VIF). All statistical procedures were performed with SPSS Statistics (version 22.0; SPSS, Inc., Chicago, IL) at an a level of 0.05 . Data in text and tables are presented as mean \pm standard deviation (SD) and as mean \pm standard error (SE) in the figures. 


\section{Chapter III - Results}

Muscle strength, force steadiness, reaction time, and pegboard times were obtained from all 27 subjects (Table 1). Comparison of one- and two-joint tests found diminished steadiness during two-joint tests. Additionally, expected positive correlations were found between similar strength and force steadiness measures. Correlation analysis found no significant associations between pegboard times and either muscle strength or reaction time. However, there were several significant correlations between measures of force steadiness and pegboard times. Multiple regression analysis, therefore, explained a moderate amount (37\%) of the variance in times to complete the Grooved Pegboard Test using a model containing a single one-joint test and two of the two-joint force steadiness tests.

\section{Pegboard Times}

Average time to compete the Grooved Pegboard Test was $52.5 \pm 4.8 \mathrm{~s}$. Pegboard times decreased through each trial, with a significant decline observed between trials one $(54.8 \pm 5.7 s)$ and two $(51.8 \pm 4.9 \mathrm{~s}, P<0.0001)$ but not between trials two and three $(50.9 \pm 5.0 \mathrm{~s}, P=0.18)$.

\section{Two-Joint Steadiness}

Consistent with previous findings, normalized force steadiness (CV) was significantly reduced at higher forces within subjects completing a similar task (e.g., pinch at $5 \%=3.92 \pm 1.44 \%$ vs. pinch at $10 \%$ $=2.63 \pm 0.89 \%, P<0.0001$ ) (Laidlaw et al., 2000). This finding was similar for each force steadiness test with the exception of index finger abduction during two-joint wrist extension-index finger abduction tests (Figure 4B).

Paired t-test comparisons of force steadiness during one- and two-joints tests found significantly less steadiness for two-joint tests $(3.78 \pm 1.23 \%$ vs. $2.50 \pm 0.71 \%, P<0.001)$ (Figure 4A). This reduction in force steadiness during two-joint tests was similar for all tests (Figure 4B). Wrist extension steadiness measured in one-joint tests $(5 \% M V C=2.35 \pm 0.81 \% ; 10 \% M V C=1.52 \pm 0.42 \%)$ was significantly $(P<$ $0.01 \& P<0.001)$ less than wrist extension steadiness measured in the two-joint tests, involving pinch and index finger abduction, respectively $(5 \% M V C=2.89 \pm 0.84 \%, 3.44 \pm 1.17 \% ; 10 \% M V C=1.94 \pm$ 
$0.45 \%, 2.50 \pm 1.29 \%)$. Pinch steadiness measured in one-joint tests $(5 \%$ MVC $=3.92 \pm 1.44 \% ; 10 \%$ MVC $=2.63 \pm 0.89 \%)$ was significantly $(P<0.01 \& P<0.001)$ less than pinch steadiness measured in the two-joint tests $(5 \% M V C=5.03 \pm 0.1 .96 \% ; 10 \% M V C=3.41 \pm 1.04 \%)$. Finally, index finger abduction steadiness measured in one-joint tests $(5 \% M V C=2.78 \pm 1.20 \% ; 10 \% M V C=1.85 \pm 0.85 \%)$ was significantly $(P<0.01)$ less than index finger abduction steadiness measured in the two-joint tests $(5 \%$

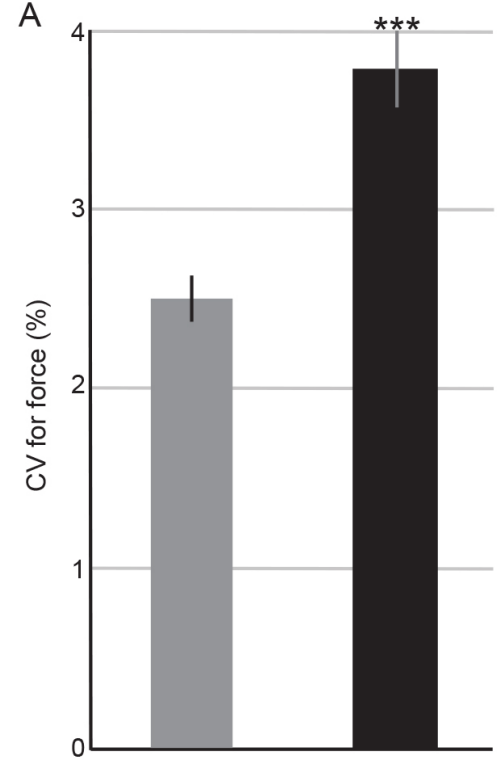

Average Steadiness
$B$
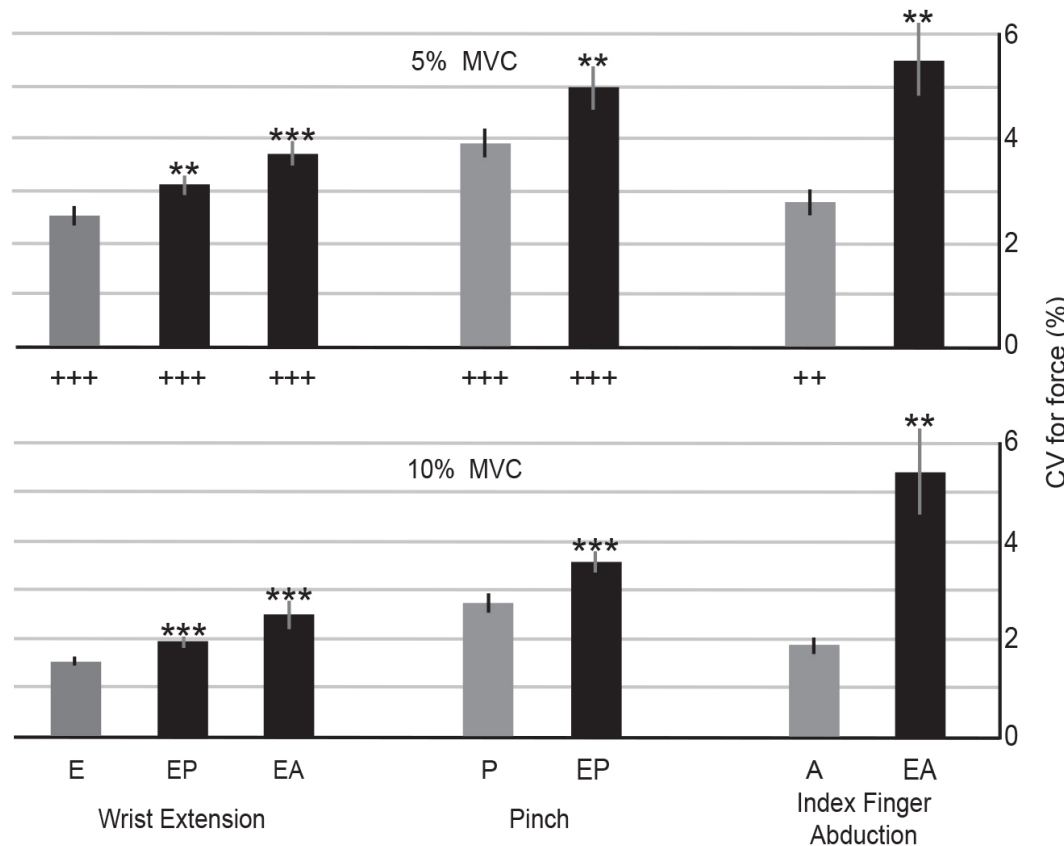

Abduction

One-Joint Test

Two-Joint Test

Figure 4. Comparison of force steadiness during one- and two-joint tests. (A) Average force steadiness across trials. (B) CV for force during each of the different tests at both $5 \%$ and $10 \%$ of MVC force. All two-joint tests were significantly less steady $\left(^{* \star *} P<\right.$ $\left.0.001,{ }^{* *} P<0.01\right)$ for all measures. $10 \% \mathrm{MVC}$ tests were significantly less steady that similar tests at $5 \%(+++P<0.001,++P<$ 0.01 ) for all measures expect index finger abduction during $E A$. $E=$ wrist extension. $P=$ pinch. $A=$ index finger abduction. $E P=$ wrist extension \& pinch. EA = wrist extension \& index finger abduction.

$M V C=5.46 \pm 3.70 \% ; 10 \% M V C=5.39 \pm 4.56 \%)$. Wilcoxon

Signed Ranks Tests confirmed the significance of these results, as steadiness values were not normally distributed.

\section{Associations Among Tests}

\section{Correlation analysis found significant associations}

among related strength and force steadiness tests.

Measures of intrinsic hand strength (pinch and index finger

abduction) as well as measures of extrinsic hand strength

A Strength

\begin{tabular}{|ll}
\hline Pinch \& Abduction & $0.480^{\star *}$ \\
Grip \& Extension & $0.857^{\star *}$ \\
\hline
\end{tabular}

B Steadiness

\begin{tabular}{|c|c|}
\hline \multicolumn{2}{|l|}{ One-Joint Test (5\%) vs. (10\%) } \\
\hline Extension & $0.787^{\star \star *}$ \\
\hline Pinch & $0.800^{* *}$ \\
\hline Abduction & $0.805^{* *}$ \\
\hline \multicolumn{2}{|c|}{ One-Joint vs. Two-Joint Test (10\%) } \\
\hline Extension \& Extension (EP) & $0.564^{* *}$ \\
\hline Pinch \& Pinch (EP) & $0.683^{* *}$ \\
\hline Extension \& Extension (EA) & $0.431^{*}$ \\
\hline Abduction \& Abduction (EA) & 0.002 \\
\hline
\end{tabular}

Table 2. Pearson correlations ( $r$ ) among tests. (A) Strength measures. (B) Force steadiness measures. $E P=$ wrist extension \& pinch. $E A=$ wrist extension \& index finger abduction. ${ }^{*} P<0.01,{ }^{*} P<0.05$. 
(grip and wrist extension) were significantly correlated $(r=0.48, r=0.86, P<0.01)$ (Table 2A). One-joint steadiness tests of wrist extension, pinch, and index finger abduction at $5 \%$ and $10 \%$ MVC force levels were strongly correlated $(r=0.79, r=0.80, r=0.81 ; P<0.01)$ (Table 2B).

Additionally, several one-joint steadiness measures were moderately correlated with steadiness during related two-joint tests. One-joint wrist extension steadiness was correlated two-joint wrist extension-pinch steadiness $(r=0.56, P<0.01)$ and with two-joint wrist extension-index finger abduction steadiness $(r=0.43, P<0.05)$. One-joint pinch steadiness was significantly correlated two-joint pinchwrist extension steadiness $(r=0.68, P<0.01)$. In contrast, one-joint index finger abduction steadiness was not correlated with two-joint index finger abduction-wrist extension steadiness $(r<0.01, P=0.99)$. Table 2B only includes correlation analysis of one- and two-joint tests at 10\% MVC; however, $5 \% \mathrm{MVC}$ correlations found similar significance. Non-parametric analysis (Spearman's correlation) confirmed the significance of these results, as steadiness values were not normally distributed.

Correlation analysis also identified associations between pegboard times and steadiness measures (Table 3). No significant associations were found between pegboard times and either reaction time $(r=-0.05, P=0.81)$ or strength $(r=-0.09, P=0.66)$. However, pegboard times were significantly correlated with force steadiness during both one-joint wrist extension at $10 \%(r=-0.45, P<0.01)$ and one-joint index finger abduction at 5\% $(r=-0.42, P<$ 0.05). Additionally, trends towards significance were

A

\begin{tabular}{lc} 
& Grooved Pegboard \\
\hline Reaction Time & -0.049 \\
Grip Strength & -0.088 \\
\hline
\end{tabular}

B

\begin{tabular}{lll} 
Steadiness Tests & $5 \% \mathrm{MVC}$ & $10 \% \mathrm{MVC}$ \\
\hline Extension & $-0.289+$ & $\mathbf{- 0 . 4 5 2 ^ { * \star }}$ \\
Pinch & -0.067 & -0.132 \\
Abduction & $-0.415^{\star}$ & -0.19 \\
| Extension & $-0.270+$ & $\mathbf{- 0 . 3 4 2}+$ \\
Pinch & -0.043 & $\mathbf{0 . 0 6}$ \\
Extension & $-0.320+$ & -0.151 \\
Abduction & -0.198 & -0.222 \\
\hline
\end{tabular}

Table 3. Pearson correlations $(r)$ between predictor variables and pegboard time. (A) No correlations were found between pegboard time and either grip strength or reaction time. (B) Significant correlations were found between pegboard performance and both one-joint wrist extension at $10 \%$ and index finger abduction at $5 \%$. Bolded variables were included in the regression model (Table 4 \& Figure 6). $E P=$ wrist extension \& pinch. $E A=$ Wrist extension \& index finger abduction. ${ }^{* *} P<0.01,{ }^{*} P<0.05,+P<0.2$.

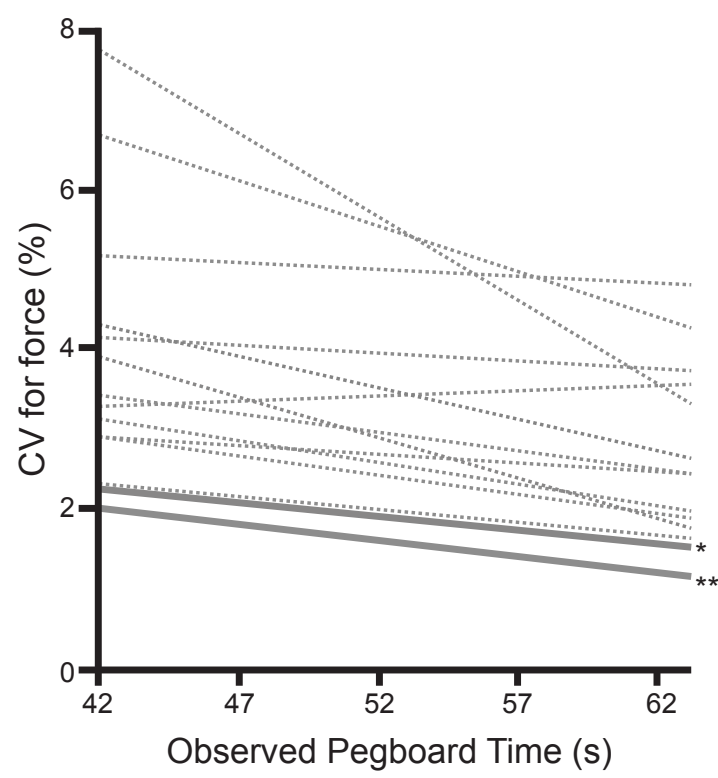

Figure 5. Regression lines highlighting negative correlations between steadiness tests and pegboard times (Table 3 ). Bolded lines identify statistically significant correlations; they include one-joint wrist extension at $10 \%$ and index finger abduction at $5 \%$. ${ }^{* *} P<0.01,{ }^{*} P<0.05$. 
identified in several force steadiness tests including: wrist extension during the two-joint wrist extensionpinch test at both $5 \%(r=-0.27, P<0.17)$ and $10 \%(r=-0.34, P<0.08)$, one-joint wrist extension at $5 \%(r$ $=-0.29, P<0.14$ ), and lastly, wrist extension during the two-joint wrist extension-index finger abduction test at $5 \%(r=-0.32, P<0.10)$. Interestingly, 13 of the 14 associations were negative, whereas the lone positive association was insignificant $(r=0.06, P=0.77)$. This indicates that individuals who were most steady tended to produce slower pegboard times. Non-parametric analysis (Spearman's correlation) confirmed the significance of these results, as steadiness values were not normally distributed.

\section{Pegboard Regression Model}

Multiple regression analysis explained $37 \%$ of the variance in times to complete the Grooved Pegboard Test using a model containing a single onejoint test and a pair of two-joint force steadiness tests $(P=0.017$, Figure 6). The three model predictors were all measured at $10 \%$ of MVC and included: wrist extension during a one-joint test, pinch during wrist extension, and wrist extension during pinch. The final regression model was: Predicted Pegboard Time $=$ $60.541-(6.177 \times$ wrist extension (one-joint) $)-(3.998$ $x$ wrist extension (two-joint) $)+(2.665 x$ pinch (twojoint)). Partial correlations and VIF measures are presented in Table 4. Negative partial correlations reflect the previously noted negative trend between steadiness and pegboard times, whereas the lone positive partial correlation indicates that increased pinch steadiness predicts a faster pegboard time.

Multiple Regression Model

\begin{tabular}{|c|c|c|c|c|}
\hline Coefficients & Beta & Sig. & Partial & VIF \\
\hline $\mathrm{E}$ & -6.177 & 0.022 & -0.465 & 1.541 \\
\hline |E & - 3.998 & 0.109 & -0.335 & 1.749 \\
\hline$P$ & 2.665 & 0.020 & 0.471 & 1.761 \\
\hline
\end{tabular}

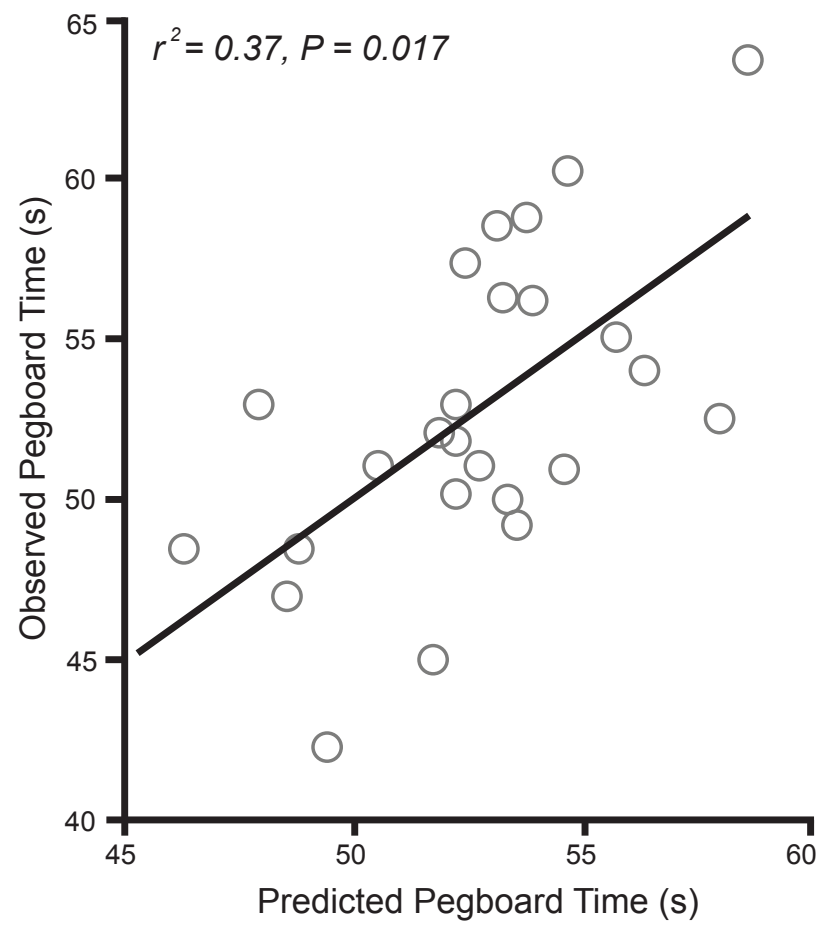

Figure 6. Multiple regression based prediction of pegboard times for young adults. Predicted times based on data from three measures of force steadiness: (1) wrist extension during a one-joint test, and combined (2) pinch and (3) wrist extension during two-joint tests.

Table 4. Multiple regression model coefficient details. $\mathrm{VIF}=$ variance inflation factor. $\mathrm{E}=$ wrist extension. $P=$ pinch. $E P=$ wrist extension \& pinch. 


\section{Chapter IV - Discussion}

The purpose of the current study was to identify predictor variables that can explain the variance in the time it takes young adults to complete the Grooved Pegboard Test. The main finding was that pegboard times were negatively associated with fluctuations in force during steady, constant-force contractions, but not with muscle strength or cognitive processing speed. Furthermore, force steadiness was worse during two-joint actions than during one-joint actions, and pegboard times were moderately predicted by a combination of force steadiness during both one- and two-joint tests.

Predicting Pegboard Performance. A re-analysis of the data for young adults from Marmon et al. (2011a) emphasizes the significance of the multiple regression analysis for the current study. The 25 young adults in Marmon et al. were similar to those in the current study in terms of age, height, body mass, pegboard time, grip strength, and gender distribution (11 females) (Table 5). Additionally, the two steadiness tests in Marmon et al. were included in the current study; these were the one-joint index finger abduction and one-joint pinch steadiness tests at $5 \%$ MVC.

\begin{tabular}{lc} 
& Total \\
\hline $\mathrm{N}$ & 25 \\
Age $(\mathrm{yrs})$ & $25.8 \pm 4.4$ \\
Height $(\mathrm{cm})$ & $170.9 \pm 11.4$ \\
Mass $(\mathrm{kg})$ & $71.5 \pm 11.9$ \\
Pegboard Time $(\mathrm{s})$ & $56.6 \pm 5.2$ \\
Grip Strength $(\mathrm{N})$ & $368.0 \pm 91.3$ \\
\hline
\end{tabular}

Table 5. Descriptive statistics of young adult subjects from Marmon et al. (2011a). Values represent mean \pm SD. Values do not significantly differ $(P>0.05)$ from the descriptive statistics in the current study (Table 1).

The regression model developed by Marmon et al. explained 36\% of the variance in pegboard times across the life span (18-89 years). The predictor variables were force steadiness during index finger abduction and grip strength. When the same regression model was applied to the pegboard times for the young adults only, the predictive power of the model was lost $\left(r^{2}=0.07, P=0.46\right)$. Similarly, application of the Marmon et al. model to the data for the young adults in the current study reveals no correlation $\left(r^{2}=0.12, P=0.85\right)$. Furthermore, re-analysis of all potential explanatory variables from Marmon et al. found no significant model able to predict the pegboard times of the young adults in that study. The strongest model approached significance and included gender, grip strength and force steadiness during the pinch grip as predictive variables $\left(r^{2}=0.28, P=0.11\right)$.

In contrast to the limited predictive power of the explanatory variables from Marmon et al., the current study was able to identify a significant model to predict the variance in pegboard times among its 
expanded set of force steadiness tests. The predictive variables, which included one-joint wrist extension at $10 \% \mathrm{MVC}$ force, two-joint wrist extension during pinch at 10\% MVC force, and two-joint pinch during wrist extension at $10 \% \mathrm{MVC}$ force, were able to explain $37 \%$ of the variance in pegboard times for the young adults. Unlike the positive correlations between force steadiness and pegboard time observed in Marmon et al., however, the correlations were almost uniformly negative in the current study. This finding indicates that increased force steadiness was associated with diminished manual dexterity, as measured by the Grooved Pegboard Test.

Negative Correlations. Despite the greater explanatory power of the regression model in the current study, the negative associations between force steadiness and pegboard time are contrary to our hypothesis. Marmon et al. identified positive associations between force steadiness and pegboard time among young, middle aged, and old adults for both pinch $(r=0.46)$ and index finger abduction $(r=0.57)$ actions. Similar trends were observed for the young adults from Marmon et al., with positive correlations for pinch $(r=0.44)$ and index finger abduction $(r=0.22)$ tasks.

The negative correlations in the current study suggest the presence of confounding variables that influence the physiological mechanisms known to be responsible for force steadiness. One potential confounder involves the speed-accuracy tradeoff in which accuracy declines with an increase in movement speed. Paul Fitts first described this speed-accuracy tradeoff quantitatively showing that movement time increases as a function of both decreasing target size and increasing movement distance (Fitts, 1954). This tradeoff represents the outcome of an optimal movement pattern in the presence of noise. The variance of this noise is proportional to the magnitude of the neural inputs received by the motor neurons. The result is fast, inaccurate movements when large forces are produced and slow, accurate movements when smaller forces are produced (Schmidt et al., 1979). Thus, it is theorized that the force steadiness and pegboard tasks of the current study are influenced by the speed-accuracy tradeoff preference of each participant. For example, individuals who tend to value accuracy above speed may produce slower pegboard times and superior force steadiness values. Conversely, individuals who tend to value speed above accuracy may produce faster pegboard times and diminished steadiness. 
Decreased Steadiness in Two-Joint Tests. Despite an equal net muscle force requirement, the observed worsening of force steadiness during two-joint actions suggests a difference in the modulation of motor unit discharge rate during the two tests. It has been shown that most of the force fluctuations during low-force isometric contractions with a hand muscle can be explained by the common modulation of motor unit discharge rate, which is in turn influenced by the visual demands of the task, type of muscle contraction, properties of the motor units recruited, and the physical activity level of the individual (Enoka et al., 2003; Negro et al., 2009). The association between force steadiness and visual feedback is underscored by previous research that observed significant reductions in force fluctuations in the absence of visual feedback. Conversely, the introduction of visual feedback was associated with increased force fluctuations (Marmon and Enoka, 2010). Therefore, differences in the visual demands associated with both the one- and two-joint tests influence the modulation of motor unit discharge rates.

Similarly, it has been shown that alteration of visual feedback can influence the magnitude of afferent feedback (Laine et al., 2014). High- gain feedback, for example, is associated with greater force fluctuations compared with low-gain feedback, decreased pre-synaptic inhibition of la terminals, and higher coherence in the discharge times for pairs of motor units. Such findings support the interpretation that more demanding visual feedback increases the drive of proprioceptive inputs to the motor neurons. Thus, changes in the neural drive to muscles may be explained by the task-related modulation of afferent feedback.

Isometric force-matching steadiness is also influenced by the presence of stressors. Exposure to a cognitive challenge, for example, increased force fluctuation when young adults perform an isometric contraction with a hand muscle, especially during fatiguing contractions (Lorist et al., 2002; Christou et al., 2004). Older adults exhibit a similar increase in force fluctuations when required to perform mental math and when receiving electric shocks (Christou et al., 2004; Vanden Noven et al., 2014). The task of controlling two forces concurrently is both physically and mentally challenging, which acts as a stressor and augments the force fluctuations.

Grip Strength. There was a wide range in grip strength $(293 \mathrm{~N}-649 \mathrm{~N})$, but this outcome was not correlated with pegboard time $(r=-0.09, P=0.66)$. Such findings contrast previously observed 
positive correlations between strength and pegboard time for young, middle-aged, and old adults studied by Marmon et al. (2011a). However, re-analysis of the Marmon et al. cohort by age group found a similar lack of correlation between strength and pegboard times for the young $(r=-0.16, P=0.22)$, middle aged $(r=0.12, P=0.57)$ and old $(r=0.05, P=0.80)$ subjects, which suggests that the relationship between strength and pegboard performance is insignificant within age groups. Other studies have also found that the time to complete a pegboard test can be improved without an improvement in grip strength (Ranganathan et al., 2001). The present findings indicate that the association between age-related declines in strength and pegboard times reported by Marmon et al. is not observed within groups.

Cognition. In contrast to the findings of Ashendorf et al. (2009) in which a set of cognitive tests was able to explain $21 \%$ of the variance in time to complete the Grooved Pegboard Test, the current study found no association between a test of cognitive function and pegboard performance. This is not particularly surprising, however, given the different tests used in the two studies. Ashendorf et al. identified associations for pegboard times with attention, executive function, and memory as tested by a Trail Making Test, the Wisconsin Card Sorting Test, and the Wechsler Memory-Visual Reproduction respectively. The current study used a reaction time test, which assesses the information processing speed of the central nervous system. However, reaction time tests are deceptively complex, and may represent more, or less about the relationship being studies than originally assumed (Salthouse and Hedden, 2002).

Conclusions. The current study demonstrated that the use of an expanded set of steadiness tests significantly improved the ability to predict pegboard times among young adults. The significant correlations were negative, however, which indicates that fast pegboard times were associated with worse force steadiness (greater CV for force values). Such findings are likely attributable to individual differences in the speed-accuracy tradeoff during the performance of the pegboard test. Despite the development of a statistically significant multiple regression model $\left(r^{2}=0.37\right)$ to predict the pegboard times of young adults in the current study, a substantial amount of the variance in the pegboard times remains unexplained and should be examined in future studies. 


\section{Chapter V - References}

Ashendorf L, Vanderslice-Barr JL, McCaffrey RJ. Motor tests and cognition in healthy older adults. Appl Neuropsychol 16: 171-176, 2009.

Backman C, Cork S, Gibson D, Parsons J. Assessment of hand function: the relationship between pegboard dexterity and applied dexterity. Can J Occup Ther 59: 208-213, 1992.

Bezdicek O, Nikolai T, Hoskovcová M, Stochl J, Brozová H, Dusek P, Zárubová K, Jech R, Ruzicka E. Grooved pegboard predicts more of cognitive than motor involvement in Parkinson's disease. Assessment (March 3, 2014).

Birznieks I. Tactile sensory control of dexterous manipulation in humans. Umeå University, Sweden. 2003.

Buchman AS, Wilson RS, Boyle PA, Bienias JL, Bennett DA. Change in motor function and risk of mortality in older persons. J Am Geriatr Soc 55: 11-9, 2007.

Christou EA, Jakobi JM, Critchlow A, Fleshner M, Enoka RM. The 1- to 2-Hz oscillations in muscle force are exacerbated by stress, especially in older adults. J Appl Physiol 97:225-235, 2004.

Clarkson PM. The effect of age and activity level on simple and choice fractionated response time. Eur $J$ Appl Physiol Occup Physiol 40: 17-25, 1978.

De Luca CJ, LeFever RS, McCue MP, Xenakis AP. Behaviour of human motor units in different muscles during linearly varying contractions. J Physiol 329, 113-128, 1982.

Enoka RM, Christou EA, Hunter SK, Kornatz KW, Semmler JG, Taylor AM, Tracy BL. Mechanisms that contribute to differences in motor performance between young and old adults. $J$ Electromyogr Kinesiol 13: 1-12, 2003.

Fitts PM. Information capacity of the human motor system in controlling the amplitude of movement. $J$ Exp Psychol Gen 47: 381-391, 1954.

Fozard JL, Vercryssen M, Reynolds SL, Hancock PA, Quilter RE. Age differences and changes in reaction time: The Baltimore Longitudinal Study of Aging. J Gerontol 49: 179-189, 1994.

Galganski ME, Fuglevand AJ, Enoka RM. Reduced control of motor output in a human hand muscle of elderly subjects during submaximal contractions. J Neurophysiol 69: 2108-2115, 1993.

Goggin NL, Meeuwsen HJ. Age-related differences in the control of spatial aiming movements. Res $Q$ Exerc Sport 63: 366-372, 1992.

Goldberg K, Faridani S, Alterovitz R. A New Derivation and Dataset for Fitts ' Law of Human Motion. J Latex Class Files 6: 1-14, 2007

Jeannerod M, Arbib MA, Rizzolatti G, Sakata H. Grasping objects: the cortical mechanisms of visuomotor transformation. Trends Neurosci 18: 314-320, 1995.

Ketcham CJ, Seidler RD, Van Gemmert AW. A, Stelmach GE. Age-related kinematic differences as influenced by task difficulty, target size, and movement amplitude. J Gerontol Ser B Psychol Sci Soc Sci 57: P54-P64, 2002. 
Kløve H. Grooved Pegboard. Lafayette, IN: Lafayette Instruments. 1963.

Laidlaw DH, Bilodeu M, Enoka RM. Steadiness is reduced and motor unit discharge is more variable in older adults. Muscle Nerve 1: 600-612, 2000.

Laine CM, Yavuz SU, Farina D. Task-related changes in sensorimotor integration influence the common synaptic input to motor neurones. Acta physiologica 211:229-39, 2014.

Logan GD. Short-term memory demands of reaction-time tasks that differ in complexity. J Exp Psychol Hum Percept Perform 6: 375-389, 1980.

Lorist MM, Kernell D, Meijman TF, Zijdewind I. Motor fatigue and cognitive task performance in humans. J Physiol 545: 313-319, 2002.

Loscher WN, Gallasch E. Myo-electric signals from two extrinsic hand muscles and force tremor during isometric handgrip. Eur J Appl Physiol 67: 99-105, 1993.

Lundergan WP, Soderstrom EJ, Chambers DW. Tweezer dexterity aptitude of dental students. J Dent Educ 71: 1090-1097, 2007.

Marmon AR, Enoka RM. Comparison of the influence of two stressors on steadiness during index finger abduction,. Physiol Behav 99: 515-520, 2010.

Marmon AR, Pascoe MA, Schwartz RS, Enoka RM. Associations among strength, steadiness, and hand function across the adult life span. Med Sci Sports Exerc 43: 560-567, 2011 a.

Marmon AR, Gould JR, Enoka RM. Practicing a functional task improves steadiness with hand muscles in older adults. Med Sci Sports Exerc 43: 1531-1537, 2011b.

Monzée J, Lamarre Y, Smith AM, Adams MJ, Johnson SA, Lefèvre P, Lévesque V, Hayward V, André T, Thonnard J, Physiol JA. The effects of digital anesthesia on force control using a precision grip the effects of digital anesthesia on force control using a precision grip. J Neurophysiol 89:672-683, 2003.

Negro F, Holobar A, Farina D. Fluctuations in isometric muscle force can be described by one linear projection of low-frequency components of motor unit discharge rates. J Physiol 587: 5925-38, 2009.

Per J, Johansson RS. Visual and somatosensory information about object shape control manipulative fingertip forces. J Neurosci 17: 4486-4499, 1997.

Ranganathan VK, Siemionow V, Sahgal V, Liu JZ, Yue GH. Skilled finger movement exercise improves hand function. J Gerontol 56: 518-522, 2001.

Reuben DB, Magasi S, McCreath HE, Bohannon RW, Wang Y-C, Bubela DJ, Rymer WZ, Beaumont J, Rine RM, Lai J-S, Gershon RC. Motor assessment using the NIH Toolbox. Neurology 80: S65-75, 2013.

Salthouse TA, Hedden T. Interpreting reaction time measures in between-group comparisons. J Clin Exp Neuropsychol 24: 858-872, 2002.

Schmidt RA, Zelaznik H, Hawkins B, Frank JS, Quinn JT. Motor-Output Variability: A Theory for the Accuracy of Rapid Motor Acts. Psychol Rev 86: 415-451, 1979. 
Semmler JG, Nordstrom MA, Wallace CJ. Relationship between motor unit short-term synchronization and common drive in human first dorsal interosseous muscle. Brain Res 767: 314-320, 1997.

Strauss E, Sherman SEM, Spreen O. A Compendium of Neuropsychological Tests. 3rd ed. New York: Oxford University Press, 2006.

Tracy BL, Enoka RM. Older adults are less steady during submaximal isometric contractions with the knee extensor muscles. J Appl Physiol 92: 1004-12, 2002.

Vanden Noven ML, Pereira HM, Yoon T, Stevens AA, Nielson KA, Hunter SK. Motor variability during sustained contractions increases with cognitive demand in older adults. Front Aging Neurosci 97:1-14, 2014.

Veale JF. Edinburgh Handedness Inventory - Short Form: a revised version based on confirmatory factor analysis. Laterality Asymmetries Body, Brain Cogn 19: 164-77, 2014.

Wang Y-C, Magasi SR, Bohannon RW, Reuben DB, McCreath HE, Bubela DJ, Gershon RC, Rymer WZ. Assessing dexterity function: a comparison of two alternatives for the NIH Toolbox. $J$ Hand Ther 24: 313-20, 2011.

Weiss A. The locus of reaction time change with set, motivation, and age. J Gerontol 20: 60-64, 1965.

Westling G, Johansson RS. Factors influencing the force control during precision grip. Exp Brain Res 53: 277-284, 1984.

Yancosek KE, Howell D. A narrative review of dexterity assessments. J Hand Ther 22: 258-69, 2009.

Young RW. Evolution of the human hand: the role of throwing and clubbing. J Anat 202: 165-74, 2003. 